Mal J Nutr 25(1): 37-45, 2019

\title{
Simple method for assessing standing height using recumbent length in bedridden patients using soft and firm mattresses
}

\author{
Wong Wei Yee ${ }^{*}$, Lim Su Lin ${ }^{1,2}$ \& Chan Yiong Huak ${ }^{3}$ \\ ${ }^{1}$ Dietetics Department, National University Hospital, Singapore; ${ }^{2}$ School of Exercise \\ and Nutrition Sciences, Queensland University of Technology, Australia; ${ }^{3}$ Biostatistics \\ Unit, Yong Loo Lin School of Medicine, National University of Singapore, Singapore
}

\begin{abstract}
Introduction: The accurate measurement of the height of bedridden patients is difficult. Height assessment is required for the calculation of body mass index, which is crucial for determining the nutrition status of a patient. This study aimed to validate recumbent length measurement against the standing height measurement using soft and firm mattresses and to derive predictive equations to calculate the actual height of bedridden patients on mattresses with different firmness. Methods: Ninety-nine hospitalised participants (mean age $48.9 \pm 13.9$ years; range $21-80$ years) (49 men, 50 women) and 100 healthy participants (mean age $36.8 \pm 13.6$ years; range $21-77$ years) ( 50 men, 50 women) were recruited. Standing height was measured using a stadiometer. Recumbent length was measured using a 2 metre long measuring tape. Hospitalised participants lay on soft mattress and healthy participants on firm mattress. Results: Using Bland-Altman plot, $96 \%$ of hospitalised participants using soft mattress were within $2.5 \pm 2.7 \mathrm{~cm}$ (mean $\pm 2 \mathrm{SD}$ ) whereas $97 \%$ of healthy participants using firm mattress were within $2.1 \pm 2.1 \mathrm{~cm}$. The regression equation developed using firm mattress was Standing height $(\mathrm{cm})=$ $0.993 \times$ Recumbent length $-0.943 ;\left(r^{2}=0.982\right)$. The regression equation developed using soft mattress was Standing height $(\mathrm{cm})=1.012 \mathrm{x}$ Recumbent length -4.477 ; $\left(r^{2}=0.981\right)$. Conclusion: We concluded that recumbent length is a valid clinical tool to estimate standing height. Standing height can be estimated from the predictive equations developed for patients lying on soft or firm mattresses.
\end{abstract}

Keywords: Recumbent length, height, measuring tape, Asian, bedridden

\section{INTRODUCTION}

Patients may be immobilised and bedridden because of stroke, head trauma, severe acute brain injury (Creutzfeldt \& Hough, 2015) or hip or leg fracture (Selikson, Damus \& Hamerman, 1988). The accurate assessment of height in this group is necessary to evaluate body mass index (BMI), which is crucial for determining the nutritional status of these patients. In addition, certain calculations of energy requirements are dependent on height, such as the Harris-Benedict formula (Harris \& Benedict, 1918) and Mifflin-St Jeor formula (Mifflin et al., 1990).

A large, multicenter study in Latin America indicated that height at

*Corresponding author: Wong Wei Yee

Dietetics Department, National University Hospital, 5 Lower Kent Ridge Road, Medical Centre,

Level 13c, Singapore 119074

Tel: (65) 67725166; Fax: (65) 67791938; Email: wei_yee_wong@nuhs.edu.sg, wongweiyee33@gmail.com doi: https://doi.org/10.31246/mjn-2018-0108 
admission was only measured in $32.9 \%$ patients (Correia \& Campos, 2003). Visual estimation of height was reported to be an inaccurate method with large potential errors (Frid et al., 2013; Bojmehrani et al., 2014). Furthermore, the self-reported height of elderly persons with poor cognitive performance has been shown to have significant errors (Sahyoun et al., 2008).

Alternative methods for length measurements of body segments to estimate height in patients with difficulty standing upright have been developed over the years. These include the knee height equation (Chumlea, Roche \& Steinbaugh, 1985; Shahar \& Pooy, 2003), demi-span equation (Hirani et al., 2010; Bassey, 1986) and arm-span equation (Shahar \& Pooy, 2003; Kwok $\&$ Whitelaw, 1991). Other studies have used ulna length (Bonell et al., 2017) and knee height (Maleki \& Shariatpanahi, 2017) as surrogates for a specific Asian country or ethnic group with subjects drawn from healthy populations and outpatients.

The use of body segment measurements is not without limitations. The measurement of knee height, demispan, arm span and ulna length require patients to be able to follow commands: knee height requires patients to be in the sitting position (Shahar \& Pooy, 2003); demi-span and arm span measurements require patients to outstretch one or both of their arms laterally (Shahar \& Pooy, 2003; Hirani et al., 2010) and ulna length is measured with patients laying their left forearm across their chest and touching their right shoulder (Bonell et al., 2017). Some bedridden patients may not be able to follow these simple commands due to their medical conditions. Moreover, these methods may not be applicable if fractures involving these body segments are present. In addition, the knee height method requires special tools such as callipers, which may not be available in many institutions.

There is growing interest in the measurement of recumbent length (RL), which is taken when lying down, to estimate the standing height ( $\mathrm{SH}$ ), which is the gold standard. RL requires less effort from patients and is easily done because it is measured in the supine position (Venkataraman et al., 2015).

Some studies have compared $\mathrm{SH}$ with the RL (Gray et al., 1985; Frid et al., 2013; Luft et al., 2008; Melo et al., 2014; Bojmehrani et al., 2014). These studies have reported that the RL overestimated the $\mathrm{SH}$; furthermore, the reported difference between the values is variable. Gray et al. (1985) found that in 108 ambulatory patients, RL was significantly longer than $\mathrm{SH}$ by $3.68 \mathrm{~cm}$. Another study by Frid et al. (2013) of 55 patients reported an overestimation of $1.9 \mathrm{~cm}$ on average compared with $\mathrm{SH}$. They stated that the advantage of RL measurement was that it was direct and gave quick results. Luft et al. (2008) compared SH in 116 hospitalised adults with RL measurements using the Luft Ruler and reported that this method overestimated height by $1.6 \mathrm{~cm}$, on the average, in patients $>60$ years of age.

A comparative analysis of methods of estimating height in 142 hospitalised patients in Brazil by Melo et al. (2014) reported that RL overestimated the actual height by $3 \mathrm{~cm}$ in men and 4 $\mathrm{cm}$ in women. A quality improvement project by Freitag et al. (2010) in 13 staff members reported that a newly designed book-end method that measured height in the supine position resulted in an overestimation of 0.5-3.0 cm compared with SH. Venkataraman et al. (2015) reported that measuring height in the supine position was easy, accurate, and reproducible, and asserted that future 
studies should focus on validating the supine method with $\mathrm{SH}$ as the gold standard.

Bojmehrani et al. (2014) evaluated 100 participants undergoing cardiac surgery to determine the accuracy of various methods in measuring the height of mechanically ventilated patients. They reported that the tape measurement method of patients lying supine was inaccurate, with a maximum error as high as $19.0 \mathrm{~cm}$. The authors did not discuss the reasons for the large error; thus far, it is the only study that reported unfavourable results using RL to estimate height.

We noted that the firmness of the mattresses that patients lay on were different. Hospitalised patients lay on soft mattress in the ward and bedridden outpatients either from home or nursing home were transferred to a firm mattress on a trolley bed in the clinic once they arrived in ambulance. Most of the aforementioned studies had not stated the firmness of the mattresses that the participants had lain on. The book-end method by Freitag et al. (2010) required participants to lie on a hard aluminium plate, but their study had a small sample size of only 13 participants. In the clinical setting, it would be impractical to transfer bedridden patients to a hard aluminium plate to measure their RL.

The measurement of RL has not been validated in the Asian population and no equations have been proposed for the local population in Singapore. The aim of this study was to validate the method of measuring $\mathrm{RL}$ using mattresses of different firmness against $\mathrm{SH}$ in Singaporeans and to develop simplified equations to estimate $\mathrm{SH}$ from $\mathrm{RL}$ for practical use in a clinical setting.

We hypothesise that RL is always more than SH and that the RL measured on soft mattress and firm mattress would differ. We have designed the study to understand these differences.

\section{MATERIALS AND METHODS}

\section{Participants}

One hundred hospitalised participants (50 men and 50 women) were recruited from the general medicine, cardiac, nephrology, oncology, neurostroke and gastro wards. Another 100 healthy participants (50 men and 50 women) were recruited from hospital staff and visitors. Hospitalised participants used soft mattress in the wards and healthy participants used firm mattress on the trolley bed in the clinic. Postulating that the accuracy of estimation of $\mathrm{SH}$ from RL was $100 \%, 100$ subjects were needed for the lower $95 \%$ CI to be $96.4 \%$. An increment in the power would have required a larger sample size which may have increased the duration of recruitment. Hence, 100 participants were recruited over a period of 2 years for soft and firm mattress, respectively.

The inclusion criteria were adults aged $\geq 21$ years and able to stand upright. The exclusion criteria were any degree of kyphosis, scoliosis, or leg and back deformities. Pregnant women were excluded for ethical reasons. The study received ethical approval from the National Health Group Domain Specific Review Board (DSRB reference: 2014/01004), and all participants provided informed written consent before participation.

The hospitalised participants were sampled from wards of different disciplines and all the participants fulfilled the inclusion criteria. We did not limit the participants to any particular ward or discipline because, eventually, we aimed to apply the equations developed for use in adult patients across all disciplines and medical conditions. On the other hand, it was challenging 
to get the same hospitalised patients to come down to clinic for RL measurement on the firm mattresses due to infection control concerns and similarly it was not practical to push the trolley bed up to ward for each RL measurement; hence healthy participants were recruited to be the subjects for the firm mattresses. Hospitalised patients were recruited to be the subjects for the soft mattresses.

The measurer, who was a dietitian who attended to patients in the ward, screened prospective hospitalised participants based on the inclusion and exclusion criteria. The potential participants were asked if they were able to stand upright and walk to the stadiometer. Consent was taken prior to measurements. The healthy participants were sourced by recruitment posters placed strategically in the lifts and in clinics that were accessible to both staff and hospital visitors. Interested potential participants telephoned the measurer to arrange the time for measurement, which was conducted during office hours. Prospective participants were screened when they arrived at clinic based on the inclusion and exclusion criteria before recruitment.

\section{Procedures}

\section{Standing height}

Standing height was measured using the SECA stadiometer, model 703 (SECA, Germany) in the wards for hospitalised participants and in the dietetics clinic for healthy participants. The participants stood facing forwards, arms hanging loosely by the side and heels against the rod, with shoes removed. The participant's back was positioned as straight as possible but not leaning on the rod (National Institute for Health Research, Southampton Biomedical Research Centre, 2014). The head was adjusted so that the Frankfort plane was horizontal (i.e., parallel to the floor). Standing height was recorded in centimetres $(\mathrm{cm})$ to one decimal point. Two measurements were taken, and the average was calculated to one decimal point.

\section{Recumbent length}

The participant lay supine on a soft mattress placed on a hospital bed for hospitalised participants or on a firm mattress on a trolley bed for healthy participants without any pillow and with feet together in a relaxed position. A hard board was placed perpendicular to the top of the head, and a point was marked using scotch tape. Subsequently, a 30$\mathrm{cm}$ ruler was placed perpendicular to the heels to obtain another point, which again was marked with scotch tape. A flexible, non-stretchable 2 metre measuring tape was used to measure the length between the two points in $\mathrm{cm}$ to one decimal point. Two measurements were taken and the average was calculated to one decimal point.

\section{Interrater reliability analysis}

Separately, another 30 healthy participants were recruited for interrater reliability analysis. Two measurers were involved in the interrater reliability measurements. Each participant had their $\mathrm{SH}$ measured twice by measurer 1 and the average was calculated to one decimal point. Then measurer 1 and measurer 2 took turns to take two readings of $\mathrm{RL}$ and the average was calculated to one decimal point. The RL readings by measurer 1 were unknown to measurer 2 during the measurement.

\section{Statistical analysis}

The data were analysed using the IBM Statistical Package for Social Sciences (SPSS) Statistics Version 24 (IBM Corp.). The Bland-Altman analysis was used to investigate the agreement between the two methods (Bland \& Altman, 1986). Two regression equations were derived to estimate $\mathrm{SH}$, one for soft mattress 
Table 1. Demographic characteristics of participants

\begin{tabular}{lcc}
\hline Variables & $\begin{array}{c}\text { Hospitalised (soft } \\
\text { mattress) } \\
n=99\end{array}$ & $\begin{array}{c}\text { Healthy (firm mattress) } \\
n=100\end{array}$ \\
\hline Gender $\quad 50(50.5)$ & $50(50.0)$ \\
$\quad$ Male [n, (\%)] & $49(49.5)$ & $50(50.0)$ \\
$\quad$ Female [n, (\%)] & $21.0-80.0$ & $21.0-77.0$ \\
Mge range (years) & $48.9 \pm 13.9$ & $36.8 \pm 13.6$ \\
\hline
\end{tabular}

SD: standard deviation

and the other one for firm mattress. The validation process is based on study done by Luft et al. (2008) and Gray et al. (1985).

\section{RESULTS}

Of the 100 hospitalised participants, one was excluded from the data analysis because she was unable to position her ankle in a relaxed manner when lying down. This was an isolated case in which the participant fulfilled the inclusion criteria: she could stand upright but when she lay down, both her ankles were plantar-flexed, causing her forefeet to be protruded. We did not encounter similar issues with the other participants. The demographic characteristics of the 100 healthy and 99 hospitalised participants are summarised in Table 1.

In hospitalised participants, the mean \pm SD of $\mathrm{SH}$ was $161.2 \pm 9.7 \mathrm{~cm}$ and the mean \pm SD of RL using soft mattress was $163.7 \pm 9.5 \mathrm{~cm}$. The $\mathrm{SH}$ and $\mathrm{RL}$ were significantly different $(p<0.001)$. On the other hand, for the healthy participants, the mean $\pm \mathrm{SD}$ of $\mathrm{SH}$ was $164.6 \pm 7.7 \mathrm{~cm}$ and the mean \pm SD of RL using firm mattress was $166.7 \pm 7.7 \mathrm{~cm}$. The $\mathrm{SH}$ and RL were also significantly different ( $p$ $<0.001$ ).

Bland \& Altman (1986) suggested that $95 \%$ of the differences between the two measurements must lie within the mean difference $\pm 2 \mathrm{SD}$ ranges. Using Bland-Altman plot, $96 \%$ of hospitalised
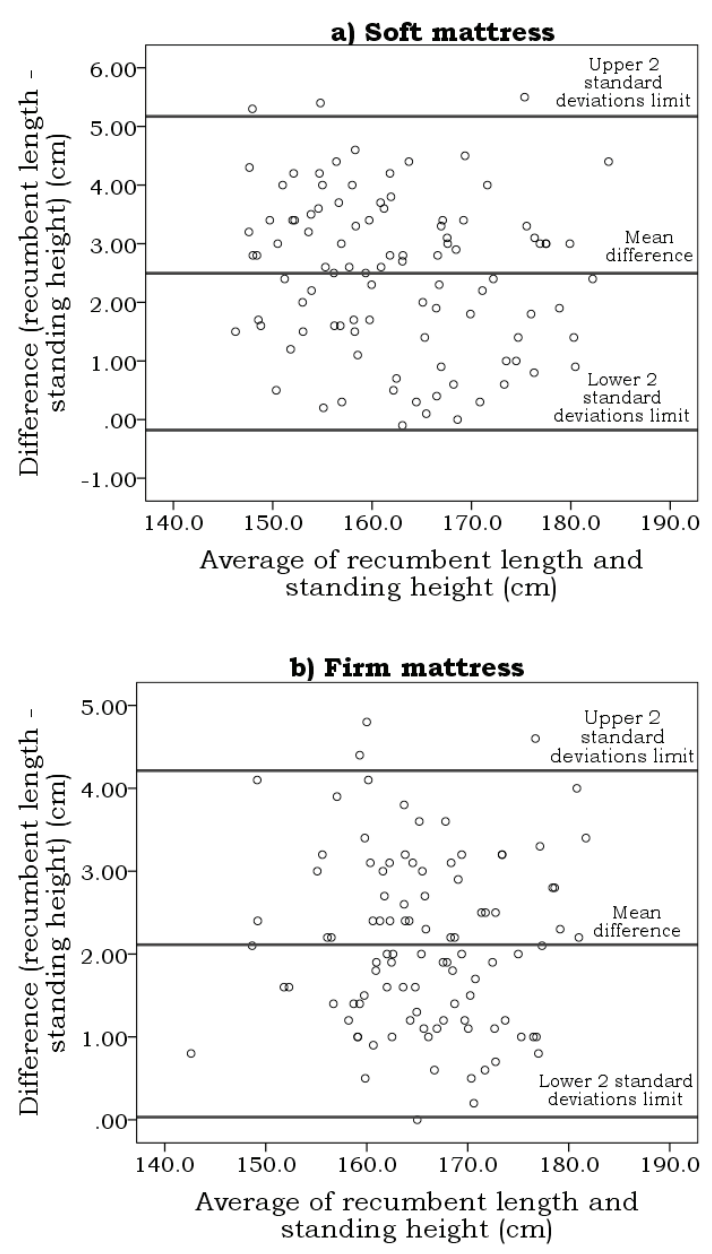

Figure 1. Bland-Altman plots for a) soft mattress and b) firm mattress. The difference in recumbent length and standing height is plotted against their average 


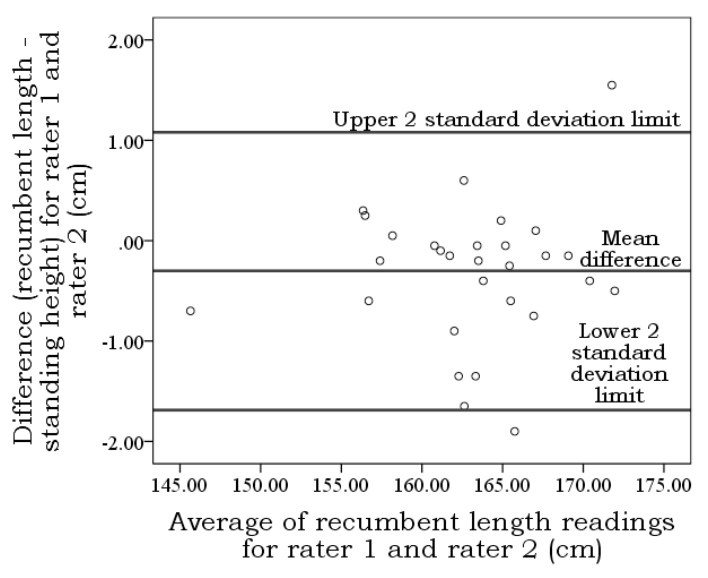

Figure 2. Bland-Altman plots for interrater reliability. The difference in recumbent length and standing height for rater 1 and rater 2 is plotted against their average

participants using soft mattress were within $2.5 \pm 2.7 \mathrm{~cm}$ whereas $97 \%$ of healthy participants using firm mattresses were within 2.1 $\pm 2.1 \mathrm{~cm}$ (Figure 1). Both results were acceptable as there were more than $95 \%$ of the participants within the mean $\pm 2 \mathrm{SD}$ ranges.

For interrater reliability analysis, the Bland-Altman plot was chosen because it shows agreement between two measurers whereas intraclass correlation (ICC) shows agreement for a group of measurers. From the BlandAltman plot, 93.3\% of the participants were within $-0.3 \pm 1.4 \mathrm{~cm}$ (Figure 2). Based on Figure 2, only two readings were out of mean \pm 2 SD but the percentage of outliers was high due to small sample size $(n=30)$.
Linear regression equations were calculated for both RLs using soft and firm mattresses and presented in Table 2. Regression equations adjusted for age and gender were developed as well given the wide age range. Using paired t-test, we compared the difference of the $\mathrm{SH}$ calculated from adjusted and unadjusted equations for both groups. The mean difference of $\mathrm{SH}$ calculated from adjusted and unadjusted equations was $0.07 \pm 0.43 \mathrm{~cm}(p=0.11)$ for soft mattress and $0.03 \pm 0.26 \mathrm{~cm}(p=0.20)$ for firm mattress. We found that there was no significant difference between the $\mathrm{SH}$ calculated from adjusted and unadjusted equations for both groups. The scatter plots between SH and RL for soft and firm mattresses are shown in Figure 3.

\section{DISCUSSION}

Measuring height can be a challenge in bedridden patients. This is also the group who need nutritional support the most (Gray et al., 1985). A reliable assessment of height is crucial for an accurate nutritional assessment, so as to deliver a tailored nutritional intervention that is specific to the patient. Many studies have been carried out in the past comparing $\mathrm{RL}$ and $\mathrm{SH}$ but none were done in the Asian population. We were interested in validating RL measurement against $\mathrm{SH}$, using simple tools such as measuring tape and clip-board in our local population and to derive equations

Table 2. Unadjusted and adjusted equations for estimating standing height using soft and firm mattresses

\begin{tabular}{lccc}
\hline Firmness & Unadjusted and adjusted equations & $r^{2}$ & Error rate $(\mathrm{cm})$ \\
\hline Soft & $\mathrm{SH}=1.012 \mathrm{RL}-4.477$ & 0.981 & -3.18 to 2.60 \\
& ${ }^{\dagger} \mathrm{SH}=0.953+0.973 \mathrm{RL}+0.007 \mathrm{~A}+1.076 \mathrm{G}$ & 0.983 & -3.15 to 2.48 \\
\multirow{2}{*}{ Firm } & $\mathrm{SH}=0.993 \mathrm{RL}-0.943$ & 0.982 & -2.71 to 2.11 \\
& ${ }^{\dagger} \mathrm{SH}=2.746+0.973 \mathrm{RL}-0.017 \mathrm{~A}+0.484 \mathrm{G}$ & 0.982 & -2.53 to 2.07 \\
\hline
\end{tabular}

RL, recumbent length; $\mathrm{SH}$, standing height; $\mathrm{A}$, age; $\mathrm{G}$, gender $(1=$ male, $0=$ female $)$

${ }^{\dagger}$ Equations adjusted for age and gender 

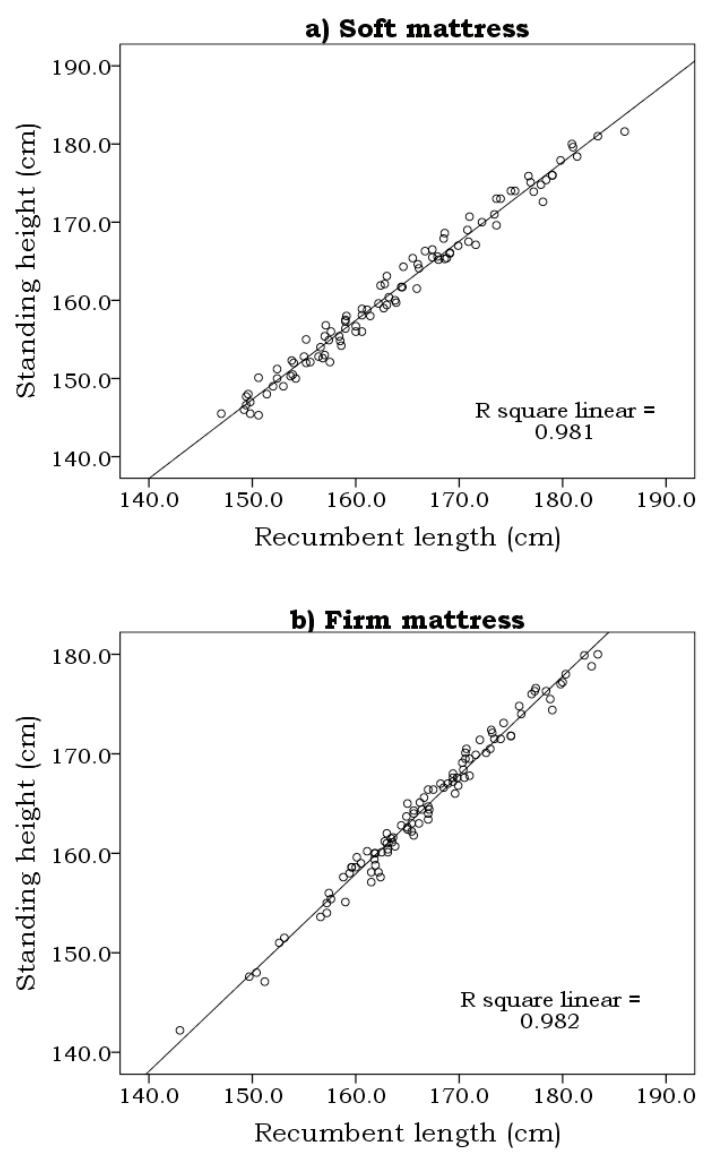

Figure 3. Scatter plots between standing height and recumbent length for a) soft mattress and b) firm mattress

to estimate $\mathrm{SH}$. We hypothesised that RL overestimated $\mathrm{SH}$ and that the readings could be different depending on the firmness of the mattress that patients lie on. These were the reasons for our study.

The present study confirmed that RL was always greater than $\mathrm{SH}$, a finding which was consistent with other similar studies (Gray et al., 1985; Frid et al., 2013; Luft et al., 2008; Melo et al., 2014). However, the aforementioned studies reported different values of overestimations of RL compared to $\mathrm{SH}$ and were carried out in non-Asians. The present study is valuable because it derived two predictive equations to estimate $\mathrm{SH}$ using $\mathrm{RL}$ on mattresses with different firmness i.e. on soft and firm, and was validated against $\mathrm{SH}$ in our local population in Singapore. To the best of our knowledge, this is the first study validating the RL equations against $\mathrm{SH}$ in an Asian population.

As reported by Gray et al. (1985), the difference observed in $\mathrm{RL}$ and $\mathrm{SH}$ is probably due to true difference of the body length and is not a consistent bias or inaccuracy in RL measurement. Keller \& Nathan (1999) reported that axial loading of the intervertebral discs occurs in static and upright postures, which compresses them and leads to height loss, whereas in recumbent postures, discs unload and regain their original height. This explains why RL is longer than $\mathrm{SH}$.

The studies discussed earlier used various tools to measure RL such as a measuring tape (Gray et al., 1985; Melo et al., 2014; Bojmehrani et al., 2014; Venkataraman et al., 2015), sliding callipers (Frid et al., 2013), Luft Ruler (Luft et al., 2008) and the book-end method (Freitag et al., 2010). Some of the tools were expensive and not easily available because they were custommade in their respective institutions. However, the measuring tape and clipboard that were used in this study are simple, inexpensive, easily available, and easy to be carried and cleaned. Therefore, our proposed measurement method is not only reproducible but also practical in routine clinical settings.

This study is the first to address the firmness of the mattress that subjects lie on during RL measurement. In this study, we observed that the firmness of mattress did affect RL and thus we included it as a variable represented by hospitalised participants for soft mattress and healthy participants for firm mattress. This study showed that the difference between $\mathrm{RL}$ and $\mathrm{SH}$ was greater when participants lay on 
a soft material. The soft mattress was observed to sink with weight causing a longer curvature. Hence two regression equations were developed to correct the $\mathrm{RL}$ for estimating $\mathrm{SH}$ in patients lying on soft and firm beds respectively. Since there was no significant difference between $\mathrm{SH}$ calculated from adjusted and unadjusted equations for both soft and firm mattresses, we decided to adopt the unadjusted equations to reduce the complexity of the equation.

Our study has several limitations. This study did not take into account the reported diurnal variation of height which has been reported to be greater in the morning and gradually reduced towards the evening (Krishnan \& Vij, 2007). In this study, $\mathrm{SH}$ was measured during office hours between $8.30 \mathrm{am}$ to $5.30 \mathrm{pm}$. The age and other characteristics of hospitalised participants were not matched and may not be comparable with that of healthy participants as the recruitment for both groups was done concurrently. Another limitation of our proposed method was that it excluded patients with contractures who were unable to straighten their legs or body. This problem was addressed by Finch $\&$ Arumugam (2014) who developed and validated a simple bedside method using measuring tape but their study was done in only 24 participants. An alternative measurement method to estimate height must be developed for this group of patients.

Future studies may consider measuring RL at a specific time of the day to minimise diurnal variation and to match the age of participants of both groups to reduce variability. The exclusion criteria should be revised to disqualify those who could not position their ankle in a relaxed manner when they lie down for measurements before recruitment. A larger sample size is needed in future research to test the interrater reliability.

\section{CONCLUSION}

We concluded that RL is a valid clinical tool to estimate $\mathrm{SH}$. Two linear regression equations were developed to estimate $\mathrm{SH}$ from RL measured on soft and firm material respectively.

\section{Acknowledgements}

We thank Ms. Christine Lin (NUH, Singapore) for assisting with the interrater reliability measurements; Dr. Loy See Ling (KK Women and Children Hospital, Singapore) for providing advice in using SPSS and guidance on writing the manuscript; and Ms. Jenny Tan (NUH, Singapore) for helping to coordinate healthy participants who arrived at the dietetics clinic for measurement. Wallace Academic Editing edited this manuscript. This study was supported by the National University Health System Allied Health and Nursing Grant (Grant number: NUHSRO/2015/054/AHN/11).

\section{Authors' contributions}

WWY, principal investigator, conceptualised and designed the study, collected and compiled the data, interpreted the data, prepared the draft of the manuscript and reviewed the manuscript; LSL, conceptualised and designed the study, interpreted the data, assisted in preparing the manuscript and reviewed the manuscript; $\mathrm{CYH}$, calculated the sample size, performed statistical analysis, interpreted the data and reviewed the manuscript.

\section{Conflict of interest}

The authors declare no conflict of interest.

\section{References}

Bassey EJ (1986). Demi-span as a measure of skeletal size. Ann Hum Biol 13(5):499-502.

Bland JM \& Altman DG (1986). Statistical methods for assessing agreement between two methods of clinical measurement. Lancet 327(8476):307-310.

Bojmehrani A, Bergeron-Duchesne M, Bouchard C, Simard S, Bouchard PA, Vanderschuren A, L'Her E \& Lellouche F (2014). Comparison of usual and alternative methods to measure height in mechanically ventilated patients: potential impact on protective ventilation. Respir Care 59(7):1025-1033.

Bonell A, Huyen NN, Phu VD, Wertheim H \& Nadjm B (2017). Determining the predictive equation for height from ulnar length in the Vietnamese population. Asia Pac J Clin Nutr 26(6):982-986. 
Chumlea WC, Roche AF \& Steinbaugh ML (1985). Estimating stature from knee height for persons 60 to 90 years of age. J Am Geriatr Soc 33(2):116-120.

Correia MITD \& Campos ACL (2003). Prevalence of hospital malnutrition in Latin America: The multicenter ELAN study. Nutrition 19(10):823825.

Creutzfeldt CJ \& Hough CL (2015). Get out of bed: immobility in the Neuro ICU. Crit Care Med 43(4):926-927.

Finch H \& Arumugam V (2014). Assessing the accuracy and reliability of direct height measurement for use in adult neurological patients with contractures: A comparison with height from ulna length. J Hum Nutr Diet 27(Suppl 2):48-56.

Freitag E, Edgecombe G, Baldwin I, Cottier B \& Heland M (2010). Determination of body weight and height measurement for critically ill patients admitted to the intensive care unit: A quality improvement project. Aust Crit Care 23(4):197-207.

Frid H, Adolfsson ET, Rosenblad A \& Nydahl M (2013). Agreement between different methods of measuring height in elderly patients. J Hum Nutr Diet 26(5):504-511.

Gray DS, Crider JB, Kelley C \& Dickinson LC (1985). Accuracy of recumbent height measurement. $J$ Parenter Enter Nutr 9(6):712-715.

Harris JA \& Benedict FG (1918). A biometric study of human basal metabolism. Proc Natl Acad Sci 4(12):370-373.

Hirani V, Tabassum F, Aresu M \& Mindell J (2010). Development of new demi-span equations from a nationally representative sample of adults to estimate maximal adult height. $J$ Nutr 140:1475-1480.

Keller TS \& Nathan M (1999). Height change caused by creep in intervertebral discs: a sagittal plane model. J Spinal Disord 12(4):313-324.

Krishnan K \& Vij K (2007). Diurnal variation of stature in three adults and one child. Anthropologist 9(2):113-117.
Kwok T \& Whitelaw MN (1991). The use of armspan in nutritional assessment of the elderly. $\mathrm{J} \mathrm{Am}$ Geriatr Soc 39(5):492-496.

Luft VC, Beghetto MG, Castro SMJ \& de Mello ED (2008). Validation of a new method developed to measure the height of adult patients in bed. Nutr Clin Pract 23(4):424-428.

Maleki SM \& Shariatpanahi ZV (2017). Estimation of stature in Iranian adults using knee height. Mal J Nutr 23(1):155-159.

Melo APF, de Salles RK, Vieira FGK \& Ferreira MG (2014). Methods for estimating body weight and height in hospitalised adults: a comparative analysis. Rev Bras Cineantropom Desempenho Hum 16(4):475-484.

Mifflin MD, St Jeor ST, Hill LA, Scott BJ, Daugherty SA \& Koh YO (1990). A new predictive equation for resting energy expenditure in healthy individuals. Am J Clin Nutr 51:241-247.

National Institute for Health Research (NIHR), Southampton Biomedical Research Centre (2014). Procedure for measuring adult height. From http: // www.uhs.nhs.uk / Media / Southampton-Clinical-Research/Procedures / BRCProcedures / Procedure-for-adult-height. pdf. [Retrieved April 17 2018].

Sahyoun NR, Maynard LM, Zhang XL \& Serdula MK (2008). Factors associated with errors in self-reported height and weight in older adults. $J$ Nutr Health Aging 12(2):108-115.

Selikson S, Damus K \& Hamerman D (1988). Risk factors associated with immobility. $\mathrm{J} \mathrm{Am}$ Geriatr Soc 36(8):707-712.

Shahar S \& Pooy NS (2003). Predictive equations for estimation of stature in Malaysian elderly people. Asia Pac J Clin Nutr 12(1):80-84.

Venkataraman R, Ranganathan L, Nirmal V, Kameshwaran J, Sheela CV, Renuka MV \& Ramakrishnan N (2015). Height measurement in the critically ill patient: A tall order in the critical care unit. Indian J Crit Care Med 19(11):665-668. 